\title{
Indonesian Stingless Bee Propolis is Effective to Accelerate Healing Process and Nutritional Status Recovery of Tuberculosis Patients
}

\author{
Ahmad Sulaeman ${ }^{1 *}$, Mahani ${ }^{2}$, Hardinsyah ${ }^{3}$ and Angelica Ploeger ${ }^{4}$ \\ ${ }^{1}$ Department of Community Nutrition, Bogor Agricultural University, Indonesia \\ ${ }^{2}$ Study Program of Food Industrial Technology, Padjadjaran University, Indonesia \\ ${ }^{3}$ Department of Community Nutrition, Bogor Agricultural University, Indonesia \\ ${ }^{4}$ Department Organic Food Quality and Food Culture, Faculty of Organic Agricultural Sciences at Kassel University, Germany \\ *Corresponding author: Ahmad Sulaeman,Department of Community Nutrition,Bogor Agricultural University,Bogor 16680, Indonesia
}

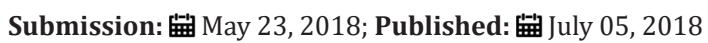

Abbreviations: ARB: Acid Resistant Bacteria; TB: Tuberculosis; ATD: Anti Tuberculosis Drug; BSLT: Brine Shrimp Lethality Test; BMI: Body Mass Index; SGPT: Serum Glutamic Pyruvic Transferase; SGOT:Serum Glutamic Oxaloacetic Tranferase; BT: Bilirubin Total; SOD: Super Oxide Dismutase

\section{Introduction}

Tuberculosis (TB) is a global disease problem and Indonesia is among of the 30 high TB burden countries in the world [1]. The standard procedure to cure TB is the patients receive antituberculosis drug (ATD) such as rifampicin. However, there are problems encountered in the use of anti-tuberculosis drugs (ATDs). One of the problems to date is their hepatotoxic properties [2] that may lead to the decreased nutritional status of patients. In fact, good nutritional status is strongly needed for the healing process.

Propolis, especially from stingless bee is a natural ingredient produced by bees and is quite abundant in Indonesia. Numerous studies [3-5] revealed that propolis has strong inhibitory ability on Mycobacterium tuberculosis (M.tbc.), has good hepatoprotective ability against toxicants and may act as immunomodulator. A study was conducted aimed to explore native stingless-bee propolis from all provinces in Indonesia and determine the best propolis as the complementary of ATDs to accelerate the healing process and nutritional status recovery [6].

\section{Phases of Study [6]}

The first phase of the study consisted of the inventory of stingless-bee apiculture in all provinces in Indonesia, identification of the cultivated stingless bee by comparing the anatomy of the tested bees with the standard anatomy and screening the propolis biological activities (antioxidant capacity, toxicity, and M.tb inhibitory activity). The antioxidant capacity test used the 1,1 Diphenyl-2-Picrylhidrazyl (DPPH) method, toxicity test used the Brine Shrimp Lethality Test (BSLT), and M.tbc. inhibition test used the Proportion method. This phase produced one propolis candidate as an ATD complementary which had strong antioxidant and hepatoprotective capability, low toxicity, and good M.tbc. inhibitory ability. In this phase, the examination of propolis's active compounds of all samples was also performed.

The second phase of the study was the clinical trial of propolis candidate as a complementary of ATDs. The study design used was a randomized controlled clinical trial (RCT), involving 50 TB patients in Bogor City that had met the inclusion and exclusion criteria. Each subject received ATD and liquid propolis package intervention for six months. The P0 group received ATD + placebo propolis package, the P1 group received a package of ATD + propolis with $6 \%$ concentration, and the P2 group received a package of ATD + propolis with $30 \%$ concentration [7].

The third phase of the study was the examination of the active compounds contained in the selected propolis candidates. The propolis samples were extracted with hydroethanolic solvent using a maceration method. The active compounds of the pure propolis extract were examined using GCMS pyrolysis. The examination was performed to identify the active compounds that played a role in inhibiting M.tbc. and acted as the hepatoprotector.

\section{Research findings}

In the first phase of the study, it was found that eight species of stingless bees has been cultivated in 10 provinces of Indonesia. A total of 14 samples of stingless-bee propolis were obtained with the following distribution: Tetragonula minangkabau (T.minangkabau) and Tetragonula moorei (T.moorei) from North Sumatera; Tetragonula laevicep (T. laevicep) from Banten; Tetragonula laevicep (T. laevicep) from West Java; Tetragonula laevicep (T. laevicep) from Central Java; Heterotrigona itama (H.itama) from West Kalimantan; Heterotrigona itama (H.itama) from East Kalimantan; Heterotrigona itama (H.itama), Geniotrigona 
thorasica (G.thorasica) and Tetragonula laevicep (T. laevicep) from South Kalimantan; Geniotrigona incisa (G.incisa) and Tetragonula biroi (T.biroi) from South Sulawesi; Tetragonula fuscobalteata (T.fuscobalteata) from West Nusa Tenggara; and Tetragonula fuscobalteata (T.fuscobalteata) from North Maluku. The G. incisa bee propolis from South Sulawesi received the highest score, and it was declared as the candidate of ATDs complement.

The results of the second phase of the study showed that the higher the concentration of propolis supplemented with ATDs, the faster the recovery which was characterized by the conversion of positive Acid-Resistant Bacteria (ARB) into negative ARB. The same pattern also occurred in the nutritional status recovery of the subjects. The supplementation of propolis to ATDs was convincingly capable of accelerating the recovery of body weight and Body Mass Index (BMI). The results of Serum Glutamic Pyruvic Transferase (SGPT), Serum Glutamic Oxaloacetic Tranferase (SGOT), Bilirubin Total (BT), Glutathione (GSH), and Superoxide Dismutase (SOD) tests also indicated that the propolis supplementation with a concentration of $30 \%$ was able to protect the liver from the hepatotoxic effect which contributed in accelerating the nutritional status recovery of the pulmonary TB patients.

In the third phase of the study, 15 active compounds were found in the selected propolis candidates. Among these active compounds, glucopyranoside compound and its derivatives were the main compounds with the concentration that reached $40.57 \%$, and they may play roles in the healing process and nutritional status recovery.

\section{Conclusion}

Various phytochemical and lipid compounds in propolis have a unique role and form a complex synergy. The combination of propolis and ATDs is ideal to speed up the healing process and nutritional status recovery. The main active compounds of the selected propolis are glucopyranoside and its derivatives. These compounds are derivatives of glucose compounds. Propolis from local stingless bee has high potency as alternative medicine to accelerate the healing process and the recovery of nutritional status of TB patients which is important to support the healing process.

\section{References}

1. World Health Organization (2017) Global Tuberculosis Report 2017.

2. Zhang ZH, Tang JH, Zhang ZL, Zhang XL, Wu, et al. (2012) Cellular toxicity of isoniazid together with rifamficin and the metabolites of isoniazid on QSZ-7701 Hepatocytes. Asian Pacific Journal of Tropical Medicine pp. 412-420.

3. Nirala SK, Bhadauria M (2008) Propolis reverses acetaminophen induced acute hepatorenal alterations: a biochemical and hispathological approach. Arch Pharm Res 31(4): 451-461.

4. Turkez H, Geyikogc F, Yousef MI, Togcar B, Vancelik S (2012) Propolis alleviates 2,3,7,8-tetrachlorodibenzo-p-dioxin-induced histological changes, oxidative stress and DNA damage in rat liver. Toxicol Ind Health 29(8): 677-685.

5. Abdelsameea AA, MAhgoub LA, Raouf SMA (2013) Study of the possible hepatoprotective effect of propolis agains the hepatotoxic effect of atorvastatin in alkbino rats. Z U M J 19(5): 112-119.

6. Mahani, Sulaeman A, Anwar F, Damanik MRM, Hardinsyah, Ploeger A (2018) Determination of Indonesian native stingless bee propolis as complementary nutraceutical candidate of anti-tuberculosis drug. Internat J Pharmacy and Pharmaceutical Science 10 (4): 15-21.

7. Kalsum N, Sulaeman A, Setiawan B, Wibawan IWT (2017) Preliminary studies of the immunomodulator effect of the propolis Trigona spp extract in a mouse model. IOSR Journal of Agric and Vet Sci 10(2): 75-80.
Creative Commons Attribution 4.0

International License

For possible submissions Click Here

\section{Submit Article}

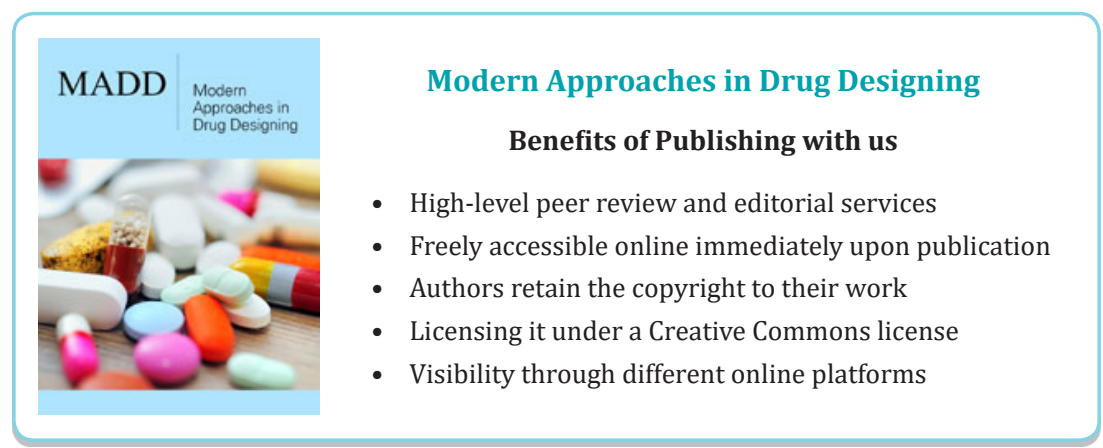

